demonstrated to be true or false but in their present forms are unsatisfactory.

(3) The hypothesis of Fredenhagen, so far as it relates to anodic oxygen or an oxygen alloy as the immediate cause of passivity, is not to be reconciled with facts.

(4) No explanation of the phenomena of passivity even approximately satisfactory has yet been produced. I have none to offer. Yet, when the experimental development warrants it, I confidently expect the cause of passivity to be found in an altered state of the metul itself, this alteration, whatever its character, being produced by any one of a number of different agencies.

In conclusion, I wish gratefully to acknowledge my indebtedness to Prof. Max LeBlanc, of the University of Leipzig, in whose laboratory this research was carried out, for his constant helpful counsel and uniform kindness during the progress of the work. I an also grateful for the courtesies and help bestowed by Dr. Bottger, Dr. Freundlich and Dr. Drucker, Privat-Dozenten in the University.

UNIVERSITY OF WASHINGTON,

July I, I908.

\title{
THE VELOCITY OF REACTIONS IN GASES MOVING THROUGH HEATED VESSELS AND THE EFFECT OF CON- VECTION AND DIFFUSION.
}

BY IRVING LANGMUIR.

Received Septenber 5, 1908.

Many of the investigations of the past few years, on the velocity of reactions in gases, have been based upon the determination of the change in the composition of the gases while passing through heated tubes. The velocity coefficient has then usually been calculated by applying the formulas derived for reactions taking place in stationary gases.

Bodenstein and Wolgast ${ }^{2}$ recently pointed out that this method of calculation is justifiable only when the gases pass through the tube entirely without mixing. For those cases where diffusion or convection has caused even partial mixing of the gases in the tube, they show that the usual method may lead to serious error. They develop formulas which hold when the mixing of the gases can be considered complete.

We have then at our disposal, for the calculation of velocity coefficients, two formulas, one of which holds when there is no mixing and the other when there is complete mixing. The only means, however, for determining which of the two formulas will give the most accurate results, in any series of experiments, is to calculate the coefficients by both formulas and then to choose that which gives the "better constant." That this method

I. phys. Chem., 6I, 422-436 (I908). 
is highly unsatisfactory became evident when Bodenstein and Wolgast applied their equations to the results obtained by Jellinek on the velocity of the reaction $2 \mathrm{NO}=\mathrm{N}_{2}+\mathrm{O}_{2}$. In one series of experiments the coefficients obtained by the new formulas were only very slightly less constant than those obtained by the old; yet the difference between the constants given by the two methods was considerable. We can also see the difficulty in using this test if we consider that the degree of mixing may be different in each of the experiments.

The object of the present paper is:

I. To obtain criteria of practical value, for deciding, in any experiment, which, if either, of the above-mentioned formulas will yield accurate results.

2. To determine, theoretically, the magnitude of the error arising from the application of the formulas to cases other than the limiting ones for which they were especially derived.

3. To develop formulas from which to calculate the velocity coefficient in those cases where neither of the above-mentioned formulas holds good, but where, in the mixing of the gases, diffusion plays a more important part than convection.

It will be the object of subsequent papers:

I. To find a means of calculating the velocity coefficient when the walls of the tube, by acting catalytically, introduce a disturbing factor.

2. To apply these theoretical results to Jellinek's ${ }^{1}$ work on the velocity of the reaction $2 \mathrm{NO}=\mathrm{N}_{2}+\mathrm{O}_{2}$; to Rowe's ${ }^{2}$ work on the velocity of combination of hydrogen and oxygen, and to the work of other investigators.

\section{General Theory of the Effect of Diffusion and Convection.}

Let us consider any reaction:

$$
n_{2} \mathrm{~A}_{1}+n_{2} \mathrm{~A}_{2}+n_{3} \mathrm{~A}_{3} \longrightarrow n_{1}^{\prime} \mathrm{A}_{1}^{\prime}+n_{2}{ }^{\prime} \mathrm{A}_{2}{ }^{\prime}+\text { etc. }
$$

We assume that the reverse reaction takes place to a negligible degree only. In accordance with the nomenclature first proposed by van't Hoff let us represent the concentration of $n_{1} \mathrm{~A}_{1}$ (not $\mathrm{A}_{1}$ !) by $c_{1}$ thus

$$
\begin{aligned}
& c_{1}=\left[n_{1} \mathrm{~A}_{1}\right] \text { or } c_{1}=\left[\mathrm{A}_{1}\right] / n_{1}, \\
& c_{2}=\left[n_{2} \mathrm{~A}_{2}\right] \text { or } c_{2}=\left[\mathrm{A}_{2}\right] / n_{2}, \text { etc. }
\end{aligned}
$$

Let us assume further that the reaction occurs without change of volume or that the volume changes only so slightly as to have a negligible effect. The necessity for this restriction is shown by Wegscheider, ${ }^{3}$ in a discussion of the influence of the changes of volume on the velocity of reactions. Applying the law of mass action we have:

${ }^{1} Z$. anorg. Chem., 49, 229-276 (1906).

${ }^{2} Z$. phys. Chem., 59, 41-7I (1907).

${ }^{3}$ Ibid., 35, 5 1 3-587 (1900). 


$$
\frac{d c_{1}}{d t}=\frac{d c_{2}}{d t}=\frac{d c_{3}}{d t}=\ldots .=-k=-k c_{1}^{n_{1}} c_{2}{ }^{n_{2}} c_{3}{ }^{n_{3}} \ldots .
$$

For the sake of simplicity we will confine our attention at present to those cases in which the mixture undergoing the reaction contains equivalent concentrations of the various constituents $A_{1}, A_{2}, A_{3}$, etc. This will not seriously restrict the usefulness of the resulting formulas. The concentrations $c_{1}, c_{2}, c_{3}$, etc., are then equal and remain equal to each other so that we may omit the subscripts. Fquation (I) thus becomes

$$
\frac{d c}{d t}=-k c^{n}, \text { where } n=n_{1}+n_{2}+n_{3}+\ldots
$$

When the change of concentration in each element of volume is caused solely by the reaction taking place in that volume, as for instance, in stationary homogeneous gas mixtures, we may integrate equation (2) directly, with the following results:

$$
\begin{gathered}
k_{\mathrm{s}} t=\log \frac{a}{c_{0}} \text { for } n=\mathrm{I} . \\
k_{s} t=\frac{\mathrm{I}}{n-\mathrm{I}}\left(\begin{array}{cc}
\mathrm{I} & \mathrm{I} \\
c_{0}^{n-\mathrm{I}} & -a^{n-1}
\end{array}\right) \text { for } n>\mathrm{I} .
\end{gathered}
$$

$k_{\mathrm{s}}$ is the velocity coefficient as calculated for stationary gases.

$a$ is the original concentration (at the time $t=0$ ).

$c_{0}$ is the concentration at the time $t$

In the case of moving gases we may apply equations (3) and (4), provided we consider our element of volume to move along with the gas and provided there is no appreciable transfer of the reacting substances, either by diffusion or convection, through the bounding surfaces of the moving element of volume. The time $t$ must be taken to mean the time required for the gas to pass the length of the tube. It was the erroneous use of equations ( 3 ) and (4) to which Bodenstein and Wolgast called attention.

When a gas mixture undergoing a reaction passes, with uniform velocity, so slowly through a vessel that difiusion and convection bring about a complete mixing of the constituents, then the concentration $c$ becomes constant throughout the vessel and becomes independent of the time. In equation (2), $d c / d t$ must then be taken to mean a partial derivative and in the integration we must consider $k c^{n}$ as constant; thus we get:

$$
a \cdots c_{0}=k_{\mathrm{M}} c_{0}^{n} t \text {. }
$$

$\mathrm{k}_{\mathrm{M}}$ is the velocity coefficient as calculated for complete mixing.

$a$ is the concentration of the reacting substance in the gas entering the vessel.

$c_{0}$ is the concentration inside the vessel and in the gas leaving the ressel.

$t$ is the average time which a particle of gas remains in the vessel. If $v$ is the volume of gas (in cc.) entering the vessel per second, measured at the temperature of the vessel, and $V$ is the volume of the vessel (in cc.), then $t=\mathrm{V} / v$. 
Equation (5) is essentially the same as those of Bodenstein and Wolgast. We have, in equations (3) and (4) and in equation (5), the two limiting cases for no mixing and for complete mixing of the gases. When $a$ and $c_{\circ}$ are nearly equal, the two formulas become practically identical, but in other cases it becomes very important to decide which formula applies most accurately.

The mixing of the gases is caused by convection currents and by diffusion.

The convection currents are produced by the inertia of the stream of gas as it enters the vessel. In such experiments as we shall consider, currents set up by differences of temperature are probably never of importance. The amount of mixing caused by convection will in general be approximately proportional to the density (inertia) of the gas and inversely proportional to the coefficient of internal friction. The latter increases with increasing temperature, approximately with the $2 / 3$ power of the absolute temperature, while the density is inversely proportional to the absolute temperature. The amount of mixing is therefore, roughly, inversely proportional to the 1.7 power of the absolute temperature. At $1100^{\circ} \mathrm{C}$. the mixing would be about $\mathrm{I} / 13$ and at $1600^{\circ} \mathrm{C}$. about $\mathrm{I} / 22$ as great as at ordinary temperatures.

On the other hand the coefficient of diffusion varies approximately with the square of the absolute temperature, so that at $I 100^{\circ}$ it is about 22 times and at $1600^{\circ}$ about $4 \mathrm{I}$ times greater than at ordinary temperatures. It is therefore highly probable that, at very high temperatures, the effect of diffusion is much greater than that of convection.

Of course convection can not well be considered mathematically and it will therefore remain a disturbing factor in all determinations of the velocity of reactions by the heated tube method. Care should be taken to design the apparatus so that convection may be avoided as much as possible.

\section{Derivation of Equations.}

Let us consider the problem of determining the effect of diffusion on the composition of a reacting gas mixture passing through a tube (Fig. I.) of which the portion $A-B$ is heated to a uniform temperature. We assume that this heated portion is sharply bounded at $A$ and $\mathrm{B}$ by thin porous plugs, in the pores of which the gases move with such high velocity that the quantity of the reacting substances carried past these points by diffusion is negligible compared

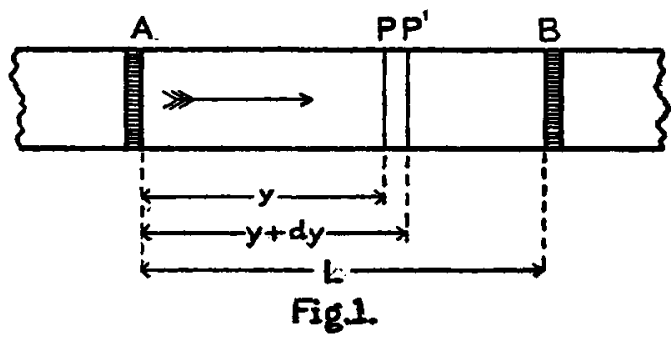


to that carried along by the movement of the gas. This condition is substantially realized in all experiments made with heated tubes or vessels, for the gas is made to enter and leave the vessel through capillary tubes, in which the velocity is so great as to render the efiect of diff usion in these tubes entirely insignificant.

We now assume that there is no mixing by convection; that is, we consider that all the parts of the gas move through the tube with the same uniform velocity, which we will designate by s.

Let us now consider the changes of concentration of one of the reacting substances $A$, at a cross-section $P$ which moves along with the gas (with the velocity s). The total change in concentration $d c$ is equal to the sum of the changes caused by time and motion respectively.

Thus,

$$
d c=\frac{\partial c}{\partial t} d t-+\frac{\partial c}{\partial y} d y
$$

but $d y / d t=s$, therefore $d y=s d t$; and $\partial c / \partial t$, the time rate of change of the concentration at any fixed cross-section of the tube, is zero, since a stationary condition is assumed to prevail. 'The equation thus reduces to

$$
d c=\cdots s \frac{\partial c}{\partial t} \cdot .
$$

In the element of volume bounded by $P$ and $P^{\prime}$ the number of mols of reacting substance $(n \mathrm{~A})$ which disappear by the reaction in the time $d t$ is according to $(\mathrm{I}): q d y d t k c_{1}{ }^{n_{1}} c_{2}{ }^{n_{3}}$. . . . where $q$ is the area of the crosssection of the tube.

Through the plane $P$, according to Fick's law, the following number of mols of $n \mathrm{~A}$ enter the element of volume:

$$
-\mathrm{D} q d t \partial c / \partial y \text {. }
$$

Here $\mathrm{D}$ is the diffusion coefficient of the gas $\mathrm{A}$. Through the section $\mathrm{P}^{\prime}$ the following amount of $n \mathrm{~A}$ leaves the element of volume;

$$
-\mathrm{D} q d t\left(\frac{\partial c}{\partial y}+\frac{\partial^{2} c}{\partial y^{2}} d y\right)
$$

The total increase in $n \mathrm{~A}$ in the time $d t$ is therefore:

Hence,

$$
q d y d t\left(\mathrm{D} \frac{\partial^{2} c}{\partial y^{2}}-k c_{1}^{n_{1}} c_{2}^{n_{2}} \ldots\right) \text {. . }
$$

$$
\frac{d c}{d t}=\mathrm{D} \frac{\partial^{2} c}{\partial y^{2}}-k c_{1}^{n_{1}} c_{2}^{n_{2}} \ldots
$$

Combining this with (6) we have:

$$
\mathrm{D} \frac{d^{2} c}{d y^{2}}-s \frac{d c}{d y}-k_{\mathrm{D}} c_{1}^{{ }^{n_{1}} c_{2}{ }^{n_{2}} \ldots . .=0} \text {. }
$$

$k_{\mathrm{D}}$ will be used to indicate the velocity coefficient calculated by considering diffusion but by neglecting convection. 
We get one such equation for each of the reacting substances $A_{1}, A_{2}, A_{3}$, etc. In order to simplify matters we are now compelled to consider only those cases in which we have equivalent quantities of the reacting substances. The equation (8) now becomes:

$$
\mathrm{D} \frac{d^{2} c}{d y^{2}}-s \frac{d c}{d y}-k_{\mathrm{D}} c^{n}=0
$$

If there is only one reacting substance or if the different substances have diffusion coefficients nearly equal, the set of simultaneous equations reduces to a single one. In many cases these conditions are fulfilled; in others we shall have to remain content with an approximation obtained by calculating the results as though all the substances had the same diffusion coefficient $D$.

Equation (9) can only be integrated, as it stands, when $n=I$. When the order of the reaction is higher than the first, let us be content for the present with approximate results; we shall see, however, that these will be very close approximations if diffusion is sufficiently active to make the difference of the concentrations, between the ends $A$ and $B$ of the tube, relatively small. We may thus put $c=c_{0}+x$ where $c_{0}$ is the concentration at the section $B$ and $x$ is a quantity small compared to $c_{\circ}$ in those cases where $n>\mathrm{I}$.

With sufficient accuracy we may write:

$$
c^{n}=\left(c_{0}+x\right)^{n}=c_{0}^{n-1}\left(c_{0}+n x\right) .
$$

Substituting this in (9) we obtain:

$$
\mathrm{D} \frac{d^{2} x}{d y^{2}}-s \frac{d x}{d y}-k c_{0}{ }^{n-1}\left(c_{0}+n x\right)=0 .
$$

This is a linear differential equation with constant coefficients and can be solved in the usual way by substituting $e^{m y}$ for $c_{0}+n x$. We then find for the complete solution:

$$
c_{\circ}+n x=\mathrm{B}_{1} e^{m_{1} y}+\mathrm{B}_{2} e^{m_{2} y}
$$

where $\mathrm{B}_{1}$ and $\mathrm{B}_{2}$ are constants of integration and $m_{1}$ and $m_{2}$ are found from the following equation by taking the + and the - signs respectively

$$
m=\frac{s \pm \sqrt{s^{2}+4 \mathrm{Dnk} k_{\mathrm{D}}{ }_{0}^{n-}} \mathrm{r}}{2 \mathrm{D}} .
$$

In order to determine the constants $B_{1}$ and $B_{2}$ we must take into account the relation existing between the diffusion coefficient, concentration, and velocity of the moving gas and the quantity (Q) of reacting substance which passes any cross-section of the tube in a given time. This relation may easily be expressed in the form of the following equation:

$$
\frac{d Q}{d t}=c q s-\mathrm{D} q \frac{d c}{d y}
$$

At a cross-section through the tube near the porous plug $\mathrm{B}(y=\mathrm{L})$ the concentration is $c_{0}$ and the amount of reacting substances passing this 
cross-section is equal to that escaping from the tube through the porous plug $\mathrm{B}$; in other words $d Q / d t=c_{0} q s$. We thus find by consideration of (I4) that for $y=I_{2}, d c / d y=0$. But $c=c_{0}+x$; hence from (12) follows:

$$
\frac{d c}{d y}=\frac{d x}{d y}=\frac{\mathrm{B}_{1} m_{1}}{n} e^{m_{1} y}+\frac{\mathrm{B}_{2} m_{2}}{n} e^{m_{2} y} \text {. }
$$

By substituting $d c / d y=0$ and $y=\mathrm{I}_{1}$ in ( $\mathrm{I}_{5}$ ) we get one of the two equations needed to determine $B_{1}$ and $B_{2}$ :

$$
\mathrm{B}_{1} m_{1} e^{m_{1} \mathrm{~L}}+\mathrm{B}_{2} m_{2} e^{m_{2} \mathrm{~L}}=0 \text {. }
$$

The other equation may be obtained by substituting $y=\mathrm{L}$ in (i2); $x$ then becomes zero by equation (10), and we have:

$$
c_{0}=\mathrm{B}_{1} e^{m_{1} \mathrm{~L}}+\mathrm{B}_{2} e^{m_{2} \mathrm{~L}} \text {. }
$$

Solving the simultaneous equations (I6) and (I7) we find:

$$
\begin{aligned}
& \mathrm{B}_{1}=\frac{-c_{0}}{e^{m_{1} \mathrm{~L}}} \frac{m_{2}}{m_{1}-m_{2}} . \\
& \mathrm{B}_{2}=\frac{+c_{0}}{e^{m_{2} \mathrm{~L}}} \frac{m_{1}}{m_{1}-m_{2}}
\end{aligned}
$$

Our next step is to find the concentrations prevailing at the end $A$ of the tube. At the porous plug $A$ the concentration will be discontinuous, that is, there will be a finite difference of concentration between the two sides of the plug no matter how thin the plug may be. I.et $a$ be the concentration of the reacting substances in the gas before passing the plug $\mathrm{A}$, and $a_{1}$ the concentration just after passing it. On the side of the plug $\mathrm{A}$ facing $\mathrm{B}$ we have $y=0$ and $c=a_{1}$, or $x=a_{1}-c_{0}$. Substituting these values in (12) we get:

$$
n a_{1}-(n-1) c_{0}=\mathrm{B}_{1}+\mathrm{B}_{2} .
$$

Although $c$ is a discontinuous function of $y$ at the point $A$, this is not the case with $d Q / d t$, for none of the reacting substance disappears within the porous plug $\mathrm{A}$. To the left of the plug, $d \mathrm{Q} / d t$ is equal to aqs, while to the right of the plug it is $a_{1} q s-\mathrm{D} q d c / d y$. Equating these two values and substituting for $d c / d y$ its value obtained from ( 15 ) by putting $y=0$, and by rearranging the terms, we find:

$$
-\frac{n s}{\mathrm{D}}\left(a-a_{1}\right)=\mathrm{B}_{1} m_{1}+\mathrm{B}_{2} m_{2} \text {. }
$$

To simplify the application of the above formulas we will define three numbers, ${ }^{1} \mathrm{P}, \mathrm{N}$ and $\mathrm{M}$, as follows:

$$
\mathrm{P}=\frac{\mathrm{SL}}{2 \overline{\mathrm{D}}} ; \quad \mathrm{N}=\frac{n \mathrm{k}_{\mathrm{D}} c_{0}{ }^{n-1} \mathrm{~L}}{s} ; \quad \mathrm{M}=\sqrt{\mathrm{P}^{2}+2 \mathrm{PN}}
$$

Then from (I3):

$$
\text { (22) }\left\{\begin{array}{l}
m_{1} \mathrm{~L}=\mathrm{P}+\mathrm{MI} \\
m_{3} \mathrm{~L}=\mathrm{P}-\mathrm{MI}
\end{array}\right.
$$

${ }^{1} \mathrm{P}, \mathrm{N}$ and $\mathrm{M}$ have each the dimensions zero. 
By substituting these in (I8) and in turn substituting the new expressions for $B_{1}$ and $B_{2}$ in ( 19 ) and by rearranging the terms we can finally make the equation take the form:

$$
n a_{1}-(n-\mathrm{I}) c_{\circ}=\frac{c_{0}}{\mathrm{M} e^{\mathrm{P}}}(\mathrm{P} \sinh \mathrm{M}+\mathrm{M} \cosh \mathrm{M}) .^{1}
$$

Similarly, from (I8), (20), (2I), and (22) we obtain:

$$
n\left(a-a_{1}\right)=\frac{c_{0} \mathrm{~N}}{e^{\mathrm{P}}} \quad \frac{\sinh \mathrm{M}}{\mathrm{M}} .
$$

To find the concentration at points between the ends $A$ and $B$, of the tube, we make these same substitutions in the equation (I2).

The result is:

(25) $\left\{\begin{aligned} c_{0}+n x & =n c-(n-\mathrm{I}) c_{0}= \\ & =\frac{c_{0}}{\mathrm{M} e^{\mathrm{P}(\mathrm{I}-\mathrm{y} / \mathrm{L})}}[\mathrm{P} \sinh \mathrm{M}(\mathrm{I}-y / \mathrm{L})+\mathrm{M} \cosh \mathrm{M}(\mathrm{I}-y / \mathrm{L}) .]\end{aligned}\right.$

By adding (23) and (24) we obtain:

$$
n a-(n-\mathrm{I}) c_{0}=\frac{c_{0}}{e^{\mathrm{P}}}\left(\cosh \mathrm{M}+\frac{\mathrm{N}+\mathrm{P}}{\mathrm{M}} \sinh \mathrm{M}\right) .
$$

We shall now consider certain special cases for which equation (26) will take very simple forms.

First Case, Mixing Nearly Complete.-Let us consider the case that $P$ is small compared to $I$, so that $P^{2}$ can be neglected in comparison with I. Then $\mathrm{M}$ is also small and $\sinh \mathrm{M}$ can be expanded into a series of which we need take only the first two terms:

$$
\sinh \mathrm{M}=\mathrm{M}+\mathrm{M}^{3} / 6 \text { and } \cosh \mathrm{M}=\mathrm{I}+\mathrm{M}^{2} / 2 .
$$

Substitute these in (26), then substitute for $M$ its value in terms of $P$ and $\mathrm{N}$ from (2I). Expand the $e^{\mathrm{P}}$ into a power series taking only the first three terms. Then divide the numerator of the second member of the equation by the denominator and neglect, in the result, terms containing $\mathrm{P}^{2}$. Rearrange the terms, substitute for $\mathrm{N}$ its value from $(2 \mathrm{I})$ and put. $\mathrm{I}=s t$, where $t$ is the time during which the gas particles remain in the tube. In this way we obtain:

$$
a-c_{\mathrm{o}}=k_{\mathrm{D}} c_{\mathrm{o}}{ }^{n} t\left(\mathrm{I}+\frac{1}{3} \mathrm{NP}\right) \text {. }
$$

This equation holds approximately in all cases where $\frac{1}{3} \mathrm{NP}$ is small (less than $0 . \mathbf{I}$ ) even if $n>\mathbf{I}$ and $a$ is large compared to $c_{0}$. For as long as NP is small, $a_{1}-c_{\circ}$ is also small compared to $c_{0}$, as can readily be shown from equation (23); and therefore (10), upon which (27) is based holds good.

By combining (5) with (27), remembering that $\frac{1}{3} \mathrm{NP}$ is small compared to $I$, we get:

$$
k_{\mathrm{D}}=k_{\mathrm{M}}\left(\mathrm{I}-\frac{1}{3} \mathrm{NP}\right) \text {. }
$$

A form sometimes more convenient can be obtained from (28) by sub-

${ }^{1} \operatorname{Sinh} \mathrm{M}$ (hyperbolic sine of $\mathrm{M}$ ) is equal to $\frac{1}{2} e^{M}-\frac{1}{2} e^{-\mathrm{M}}$; $\cosh \mathrm{M}=\frac{1}{2} e^{\mathrm{M}}+\frac{1}{2} e^{-\mathrm{M}}$. (See J. W. Mellor's Higher Mathematics, Etc., for a table of the hyperbolic functions.) 
stituting for $\mathrm{N}$ and $\mathrm{P}$ their values from (21). Then by considering that $k_{\mathrm{M}}$ and $k_{\mathrm{D}}$ differ from each other only slightly and that $\mathrm{L}_{\mathrm{s}}=s t$, and by combining with $(5)$ we get:

$$
k_{\mathrm{D}}=k \quad\left(\mathrm{I}-\frac{n s \mathrm{I}}{6 \mathrm{D}} \frac{a-c_{0}}{c_{0}}\right) .
$$

Second Case, Only Slight Mixing.-We will now take the case where $\mathrm{D} / \mathrm{s}$ is so small that there is only slight mixing of the gases in the tube. The concentration $a_{1}$ therefore, will differ only little from $a$ so that if $a$ is large compared to $c_{\circ}$ the equation (26) will not hold except when $n=\mathrm{I}$. Hence we can best treat the case before us by going back to the original differential equation (9). Since $D$ is small we can, without serious error, substitute for $d^{2} c / d y^{2}$ an approximate value obtained as follows. Put $\mathrm{D}=\mathrm{o}$ in (9); this gives us a sufficiently accurate value for $d c / d y$. By differentiation we get:

Substitute this in (9):

$$
\frac{d^{2} c}{d y^{2}}=-\frac{n k_{\mathrm{D}} c^{n-1}}{s} \frac{d c}{d y}
$$

$$
\frac{\mathrm{D} n k_{\mathrm{D}}}{s} \frac{d c}{c}+s \frac{d c}{c^{n}}=-k_{\mathrm{D}} d y \text {. }
$$

This equation holds between the porous plugs $A$ and $B$, but since $c$ is discontinuous at $\mathrm{A}$, it does not apply at $\mathrm{A}$ itself.

$k_{\mathrm{s}}$ can be calculated from (30) by placing $\mathrm{D}=0$ :

$$
s \frac{d c}{c^{n}}=-k_{\mathrm{s}} d y
$$

Subtract this from $(30)$ :

$$
\left(k_{\mathrm{D}}-k_{\mathrm{s}}\right) d y=\frac{\mathrm{D} n k_{\mathrm{D}}}{s} \frac{d c}{c} .
$$

Since $k_{\mathrm{D}}-k_{\mathrm{s}}$ is small we can substitute $k_{\mathrm{s}}$ for $k_{\mathrm{D}}$ in the second member, making an error of the second order only. We now integrate: for $y$ between the limits o and L, and for $c$ between the limits $a_{1}$ and $c_{0}$.

$$
\left(k_{\mathrm{D}}-k_{\mathrm{s}}\right) \mathrm{L}=\frac{\mathrm{D} n k_{\mathrm{s}}}{s} \log \frac{a_{1}}{c_{0}} .
$$

$a_{1}$ differs from $a$ by a small quantity of the first order, but in determining $k_{\mathrm{D}}-k_{\mathrm{s}}$ we can replace, in the second member, $a_{1}$ by $a$ and cause an error of the second order only. The equation may now be written:

$$
k_{\mathrm{D}}=k_{\mathrm{S}}\left(\mathrm{I}+\frac{\mathrm{D} n}{s \overline{\mathrm{L}}} \log \frac{a}{c_{\mathrm{o}}}\right) \text {. }
$$

When $n=\mathrm{I}$ or when $a$ does not differ much from $c_{0}$ equation (3I) can be shown to be equivalent to:

$$
k_{\mathrm{D}}=k_{\mathrm{s}}\left(\mathrm{I}+\frac{\mathrm{N}}{2 \mathrm{P}}\right)
$$

This last result can also be obtaines from (26) by methods somewhat 
analogous to those used in the derivation of (27), making the assumption that $D$ is small and neglecting terms of the second order.

We see by a consideration of the equations $(32),(28)$ and $(2 I)$ that $I / P$ is a measure of the amount of mixing caused by diffusion.

When I/P is zero there is no mixing and equation (26) reduces to (3) or (4) or at least it does so when the assumption ${ }^{1}$ that was made in its derivation, is fulfilled.

When I $\mathrm{P}$ increases without limit then there is complete mixing and equation (26) becomes identical with (5).

\section{Conclusions and Summary. ${ }^{2}$}

In order that the formulas which have been derived, may be applied by those who have not cared to follow the rather long calculations that were necessary, the following pages will be devoted to a brief statement of the problem which I have attempted to solve and of the results obtained.

We have considered the problem of determining the change of composition which will occur in a reacting gas while it passes with uniform velocity through a heated tube. We have taken into account the effect of diffusion and convection on such change of composition.

The heated portion of the tube is assumed to have a uniform temperature and to be of the same cross-section throughout its length. The gases are supposed to enter and leave this heated portion so rapidly that no perceptible reaction takes place except in this sharply defined heated region. This may be done in practice by using capillary tubes.

We consider only those cases where the walls of the tube do not act catalytically and where the reaction takes place without change of volume or where the change of density in passing through the tube is small-in any case, less than ten per cent.

If the reaction is of an order higher than the first, that is, if $n>I$ we assume:

I. That the reacting substances are present in equivalent amounts.

2. That the diffusion coefficients of the different reacting substances have the same magnitude. If this should not be the case, then those formulas given below which involve the diffusion coefficient $D$ will not give accurate results; but in most cases by substituting in the formula first the value of the largest diffusion coefficient possessed by any of the substances and then the value of the smallest coefficient, we can obtain two results between which the true result must lie. It should be noted that the diffusion coefficients of gases do not differ very largely from each other.

1 This assumption is that when $n>1, a_{1}$ differs only little from $c_{0}$.

2 For the meaning of the symbols used see the list at the end of this paper. 
In the following several paragraphs, are given the formulas of the most practical value.

$$
k_{\mathrm{s}} t=\log \frac{a}{c_{0}} \text {. This holds only for } n=\mathrm{r} .
$$

$$
k_{\mathrm{s}} t=\frac{\mathrm{I}}{n-\mathrm{I}}\left(\frac{\mathrm{I}}{c_{0}^{n-1}}-\begin{array}{c}
\mathrm{I} \\
a^{n-1}
\end{array}\right) \text {. This holds for } n>\mathrm{I} \text {. }
$$

These formulas hold accurately only when diffusion and convection do not cause any perceptible mixing of the gases in the tube.

If it can be safely assumed that convection plays but a small part compared to diffusion-and from the considerations given in the early part of this paper (after equation 5) this does appear probable-then we may say with certainty that equations (3) and (4) hold accurately whenever the quantity $\frac{n \mathrm{D}}{s L} \log \frac{a}{c_{0}}$ is negligibly small compared to $\mathrm{I}$. If this quantity is not negligible but has a value less than $\mathrm{O}$. I, then the following equation gives a very close approximation to the true velocity coefficient:

$$
k_{\mathrm{D}}=k_{\mathrm{s}}\left(\mathrm{I}+\frac{n \mathrm{D}}{s \mathrm{~L}} \log \frac{a}{c_{\mathrm{o}}}\right) \text {. }
$$

When diffusion and convection are so active as to cause complete mixing of the gases in the tube, the velocity coefficient should be calculated from the following equation:

$$
a-c_{0}=k_{\mathrm{M}} c_{0}{ }^{n} t \text {. }
$$

In order to decide whether the mixing is complete or not, we have the following simple criterion. If the quantity $\frac{n s \mathrm{~L}}{6 \mathrm{D}} \frac{a-c_{0}}{c_{0}}$ is negligible compared to $I$, then it is certain that the mixing is so nearly complete that equation (5) will hold accurately. When this quantity is not negligible but has a value not exceeding $o$. I then the following is a close approximation for the velocity coefficient if convection plays but a small part compared to diffusion:

$$
k_{\mathrm{D}}=k_{\mathrm{M}}\left(\mathrm{I}-\frac{n s \mathrm{~L}}{6 \mathrm{D}} \frac{a-c_{0}}{c_{0}}\right) .
$$

If we are not able to assume that the effect of convection is negligible, we have, at least, the following to help us to determine the true velocity coefficient $k$ :

(33)

$$
k_{\mathrm{s}}<k_{\mathrm{D}}<k<k_{\mathrm{M}} \text {. }
$$

When convection is negligible $k$ becomes identical with $k_{\mathrm{D}}$. On the other hand when convection is much more important than diffusion, $k$ approaches $k_{\mathbf{M}}$.

Equations (3I) and (29) should never be applied except when the second terms in the parenthesis are numerically less than 0.1 or (for 
very rough results) 0.2 . In those cases where both these quantities are larger than the numbers given, the coefficient $k_{\mathrm{D}}$ may be calculated with almost any desired degree of accuracy, by a method of approximation, from equation $(26)^{1}$ provided that when $n>$ I the quantity $a_{1}$ (calculated from equation 23) differs only by a relatively small amount from $c_{0}$. The details of this calculation will be discussed later in a paper which will deal with the application of the formulas here derived.

The numerical values of the diffusion coefficients of many pairs of gases are easily available, for example in Landolt-Börnstein's Tables; in all cases, however, the diffusion coefficients may be calculated with sufficient accuracy from the coefficients of internal friction. ${ }^{2}$ The order of magnitude of all diffusion coefficients is about the same. In nearly all cases, except when one of the gases is hydrogen, the diffusion coefficients have values at $0^{\circ} \mathrm{C}$. which lie between 0.09 and $0.2 \mathrm{~cm} .{ }^{2}$ per second. The diffusion coefficient increases rapidly with the temperature, nearly in proportion to the square of the absolute temperature.

In conclusion, to give a clearer idea of the effect of diffusion on the composition of gases passing through heated tubes, I have calculated, as an example, a curve (Fig. 2 ) giving the concentration of the reacting substance in a hypothetical experiment as a function of the distance from $A$, the end of the tube at which the gas enters.

The gas is assumed to enter the tube (of Io cm. length) with the concentration $a=22.32$ and to have the velocity $s=\mathrm{I} \mathrm{cm}$. per second while in the tube. The reaction is taken to be monomolecular and its velocity coefficient equal to $\mathrm{O} . \mathrm{I}$. Three cases are considered:

I. Diffusion and convection play no part; this gives us the curve $S$, calculated from equation (3).

2. Convection is absent, but diffusion occurs, the value of $\mathrm{D}$ being taken as equal to 5.0. Curve $D$ is thus calculated from equations (25) and (24).

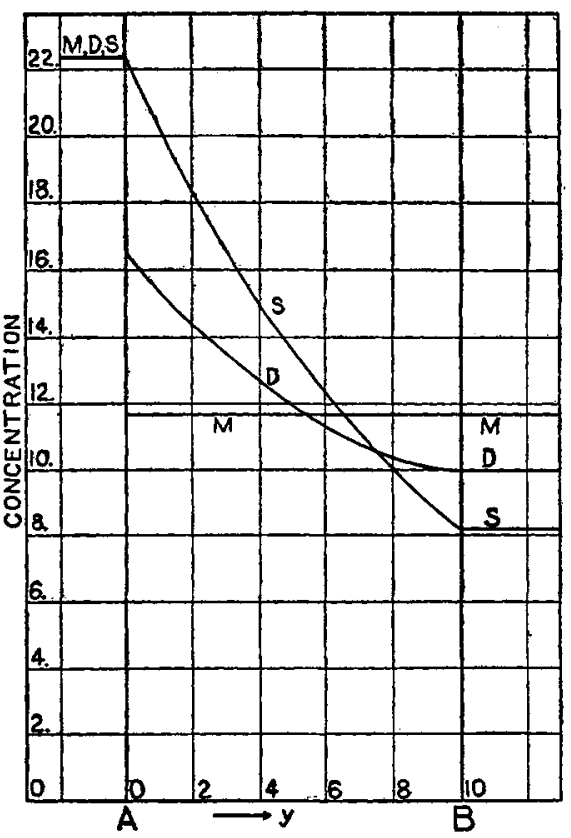

Fig. 2 .

${ }^{1}$ See under the heading Derivation of Equations.

${ }^{2}$ See O. E. Meyer, Die Kinetische Theorie der Gase, 2nd Ed., pp. 274. 
3. Diffusion and convection cause complete mixing, thus making the concentration throughout the heated portion of the tube constant. Equation (5) here gives us the curve M.

It appears, clearly, from the curves that difiusion and convection tend to decrease $a-c_{0}$, that is, they act in a way equivalent to a decrease in the velocity of the reaction.

$$
\text { List of Symbols and Their Meanings. }
$$

The numbers in parentheses refer to equations in the text.

$a=$ concentration of the reacting substances in the gases before entering the heated portion of the tuhe. See $c$.

$a_{1}=$ concentration of the reacting substances immediately after entering the heated portion of the tube. Thus in Mig. I, $a_{1}$ is the concentration on the right-hand side of the porous plug $A$. See $c$.

$c=$ concentration of the reacting substance inside the heated portion of the tube, at any distance $y$ from the porous plug A, Fig. I. All concentrations are to be measured in mols. per unit of volume.

$c_{0}=$ concentration of the reacting substances in the gases leaving the heated portion of the tube. See $c$.

$k=$ true velocity coefficient of the reaction. Defined by (2) when this is applied to homogeneous gas mixtures.

$k_{\mathrm{D}}=$ velocity coefficient calculated from (26), (29), (3I) or others. It may be different from $k$.

$k_{M}=$ velocity coefficient calculated from (5); it may differ from $k$.

$k_{\mathrm{s}}=$ velocity coefficient calculated from (3) or (4); it may differ from $k$.

$\mathrm{L}=$ length of heated portion of tube.

$\mathrm{M}=\mathrm{a}$ number defined by $(2 \mathrm{I})$.

$\mathrm{N}=$ a number defined by (21).

$n=$ order of the reaction. See (2).

$\mathrm{P}=$ a number defined by $(2 \mathrm{I}) ; \mathrm{I} / \mathrm{P}$ is a measure of the amount of mixing caused by diffusion.

$s=$ linear velocity of the gases while passing through the heated portion of the tube.

$t=$ time required for the gases to pass the length of the heated portion of the tube. $t=\mathrm{L} / \mathrm{s}$.

$y=$ distance measured to the right from the porous plug A, Fig. I.

MORTON Memortal Laboratory, StrVens Institute,

HOBOK EN, N. J. 